\title{
The labio-mandibular flap for upper lip and peri- commissural defects
}

\author{
Ruben Yap Kannan, Charles Nduka \\ Department of Plastic Surgery, Queen Victoria Hospital, East Grinstead RH19 3DZ, UK.
}

Correspondence to: Dr. Ruben Yap Kannan, Department of Plastic Surgery, Queen Victoria Hospital, East Grinstead RH19 3DZ, UK. E-mail: ruben.kannan@gmail.com

How to cite this article: Kannan RY, Nduka C. The labio-mandibular flap for upper lip and peri-commissural defects. Plast Aesthet Res 2018;5:4. http://dx.doi.org/10.20517/2347-9264.2018.02

Received: 8 Jan 2018 First Decision: 24 Jan 2018 Revised: 26 Jan 2018 Accepted: 27 Jan 2018 Published: 31 Jan 2018

Science Editor: Raúl González-García Copy Editor: Jun-Yao Li Production Editor: Cai-Hong Wang

\begin{abstract}
Aim: Peri-commisural defect reconstruction using the Abbe or Estlander flaps tend to pilfer tissue from the lower lip, contributing to microstomia, with its attendant problems. In this study, we aim to design a flap for more superficial defects, in which the underlying orbicularis oris muscle can be preserved when resecting peri-commisural skin malignancies whilst also ensuring completeness of excision.
\end{abstract}

Methods: In a retrospective case review of 7 cases at our institution over a 12-month period (2016-2017), we conceptually designed a perforator-plus fascio-cutaneous flap from within the labio-mandibular fold with a 6-month follow-up in terms of oncological clearance and aesthetic outcome. The cohort was composed of patients with skin cancers e.g. basal and squamous cell carcinomas, presenting to a tertiary care facial plastic surgery centre. The technique involved raising a flap from within the peri-oral area, with a scar disguised along the labio-mandibular and naso-labial folds which allows for both an aesthetic reconstruction and the preservation of the oral sphincter mechanism, by avoiding microstomia. The outcomes measured were (1) to ascertain whether this procedure is oncologically safe, (2) there were instances of microstomia and (3) aesthetic appearance.

Results: All oncological lesions were completely excised in all cases and at up to six months' follow-up, there were no instances of recurrence. Functionally, oral sphincter function was preserved in all instances as was aesthetic appearance.

Conclusion: The labio-mandibular flap is an oncologically safe procedure for skin cancers whilst preserving oral sphincter function and maintaining aesthetics. It is hence, a superior alternative to Abbe and Estlander flaps, for more superficial defects, not requiring mucosal excision.

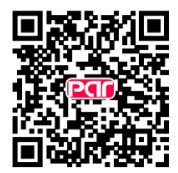


Keywords: Peri-commisural reconstruction, Abbe flap, Estlander flap, labio-mandibular flap

\section{INTRODUCTION}

Upper lip reconstruction is a surgical challenge due to the high level of lip anatomy detail. This has led to the development of a "like for like" reconstruction using the "Abbe" or "Estlander" flap for upper lip and commissural defects respectively. The latter flap has the disadvantage of causing microstomia, while the Abbe flap has the additional hassle of being a two-stage lip-switch technique. In the vast majority of these cases, the primary indication is post-oncological reconstruction e.g. following skin cancer excisions. This brings to bear the next question, i.e. do these tumours necessitate full-thickness lip excisions, as they rarely involve oral mucosa.

An alternative reconstructive option here would be the use of perforator flaps for partial-thickness defects. One option to consider here would be the superior labial artery perforator flap for upper lip defects ${ }^{[1]}$. However, as with islanded flaps, they tend to develop lymphoedema and patients are often left with an unaesthetic appearance. Leaving a small skin bridge should reduce this risk but this cannot be done in a conventional manner as the length:width ratio philosophy will not allow for it. However, with the application of the perforator-plus concept ${ }^{[2]}$, such a combination is possible.

In this article, we describe the use of flaps based on a combination of traditional aesthetic sub-units; the labio-mandibular fold (LMF) and the naso-labial fold but tempered with the perforator-plus concept for an ideal blend of "beauty and blood supply".

\section{METHODS}

In a retrospective case review over 12 months at our institution (2016-2017), we performed seven cases of upper lip reconstruction $(n=7)$, all as part of post-oncological reconstruction. These defects were reconstructed using a rotation-advancement flap in the supra-muscular plane, raised from within the skin of the LMF with sparing of the vermilion border of the lip. Just lateral to the oral commissure, a musculo-cutaneous perforator, arising from the modiolus is consistently found and preserved. Once this modiolar perforator is identified, the entire flap is advanced into the defect to close the primary defect first. As the flap then overlaps the oral commissure, a Y-V advancement of the oral commissure is performed, just proximal to the modiolar perforator, to translate the angle of the mouth laterally and re-establish the aesthetics of the oral commissure. This corrects microstomia and re-establishes lip aesthetics as graphically illustrated in Figure 1.

\section{Case illustrations}

An 80 -year old lady presented with a $15 \mathrm{~mm} \times 10 \mathrm{~mm}$ superficial basal cell carcinoma of the right upper lip [Figure 2], which was excised with part of the orbicularis oris and preservation of the oral mucosa. This resulted in a $23 \mathrm{~mm} \times 18 \mathrm{~mm}$ defect over the upper lip, which was reconstructed with a labio-mandibular flap. As shown in Figure 3, the outer border of the flap is along the LMF whilst the inner margin is at the vermilion border of the lip. The "length:width" ratio of the flap is approximately 4:1 and once inset, sets in seamlessly along the aesthetic lines of the lip and the LMF. Once healed, the scars are hardly discernible as they sit along the wrinkle lines of the upper lip and the LMF, as shown in Figure 4.

The usefulness of the labio-mandibular flap is illustrated once more in another case wherein a large skin tumour, as is shown in Figure 5, involved 40\% of the upper lip with infiltration of the orbicularis oris beneath the tumour. Conventionally, this would have required a wedge excision and reconstruction with an Abbe flap, in two stages. Following excision of the lesion with a smaller section of the orbicularis oris, a labiomandibular flap was raised and advanced into the defect [Figure 6]. Postoperative images [Figure 7] show 


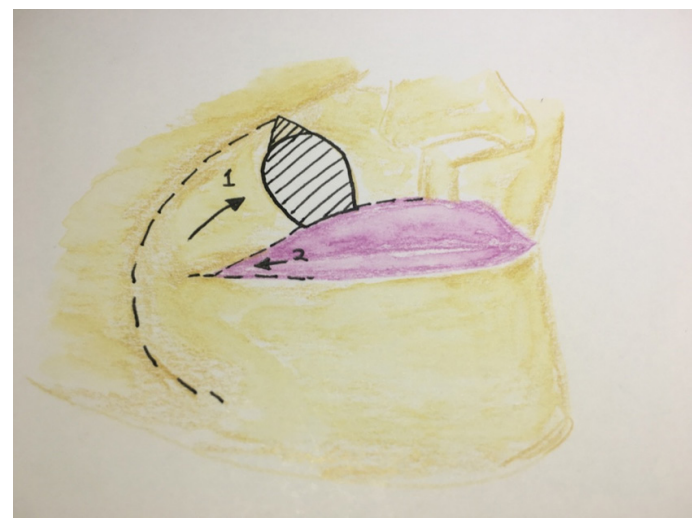

Figure 1. A graphical illustration of the labio-mandibular flap raised from the peri-oral and lower lip areas, along the LMF and vermilion border, illustrated by " 1 ". Note also the Y-V advancement of the oral commissure into the flap itself (illustrated as " 2 "). The presence of the orbicularis oris musculocutaneous perforator within the distal flap ensures its continued perfusion while the narrow cutaneous bridge at the oral commissure suffices for venous and lymphatic return

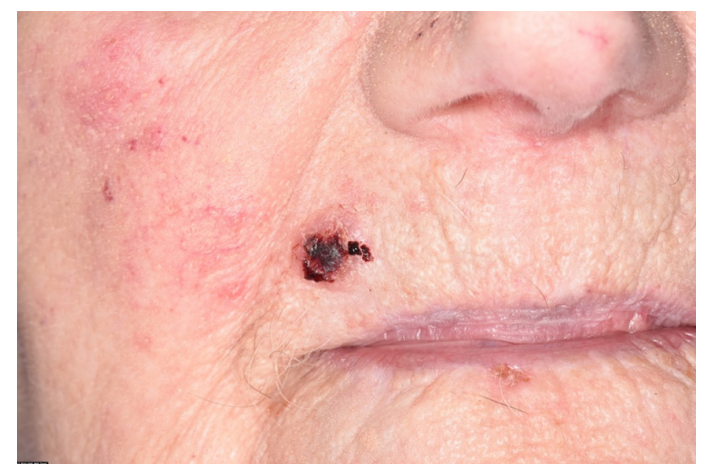

Figure 2. A large superficial basal cell carcinoma over the upper lip/commissural area

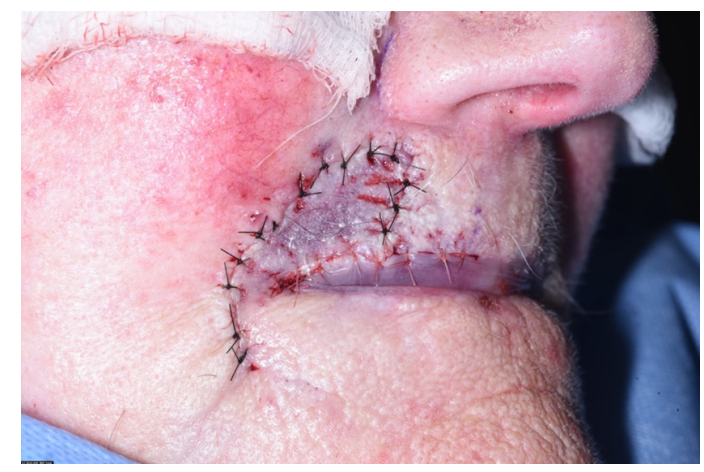

Figure 3. Final post-operative results of the labio-mandibular flap on the patient

the excellent lip symmetry at rest with a completely normal functioning orbicularis oris. Note the normal dimensions of the oral aperture, the absence of microstomia as well as the seamless healing.

\section{RESULTS}

There were 7 patients' in our cohort with 6 females and 1 male patient. All patients were over the age of 60 years. The mean defect size was $23 \mathrm{~mm} \times 22 \mathrm{~mm}$, following oncological resections of skin cancers. On reconstructing with the LMF flap, all flaps survived completely, with healing achieved within a week at the 


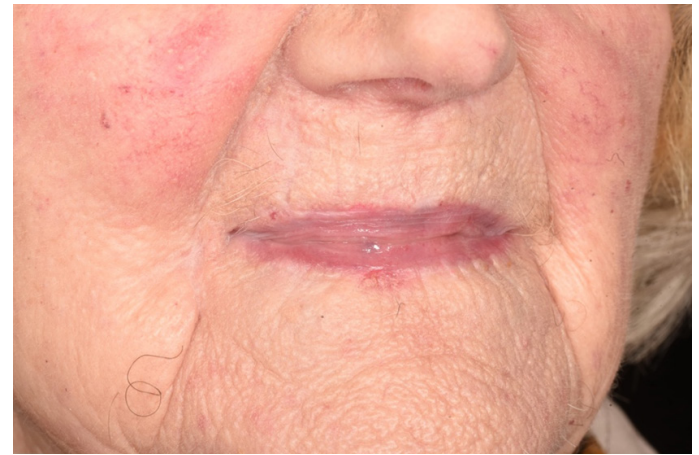

Figure 4. An image of the same patient, showing a seamless aesthetic appearance, at 3 months' postoperation

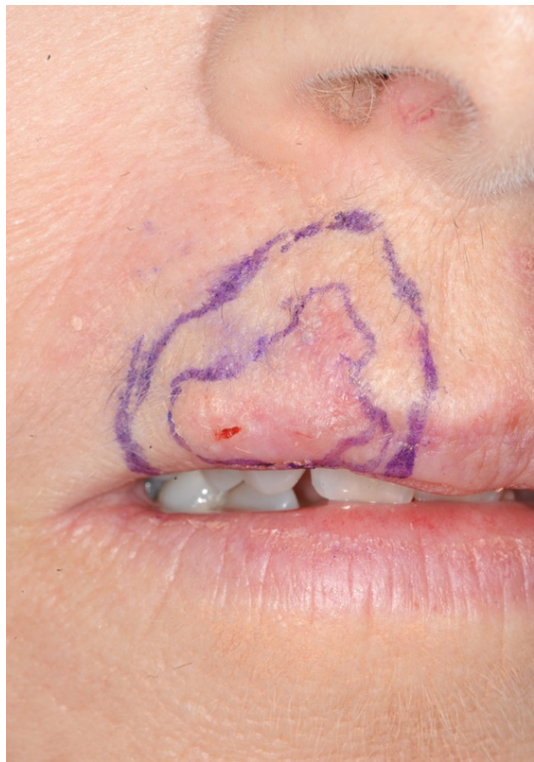

Figure 5. Preoperative image of a large skin cancer involving $40 \%$ of the width of the upper lip and extending past the vermilion border, onto the lip itself

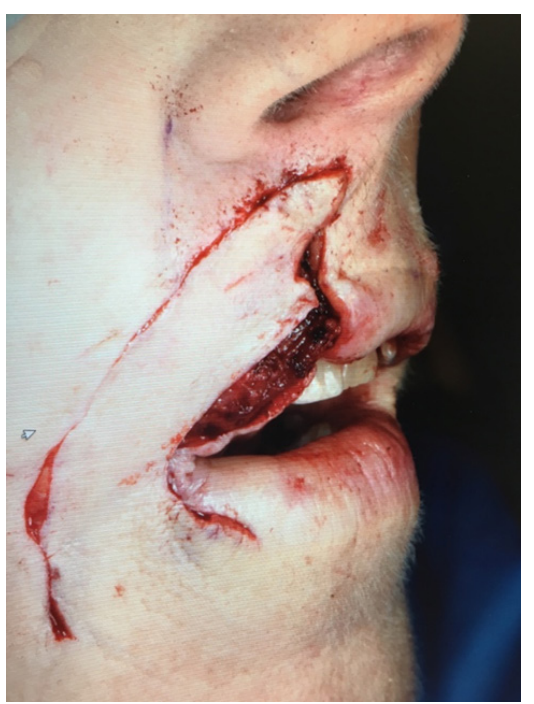

Figure 6. Intra-operative image of labio-mandibular flap being advanced into the defect 

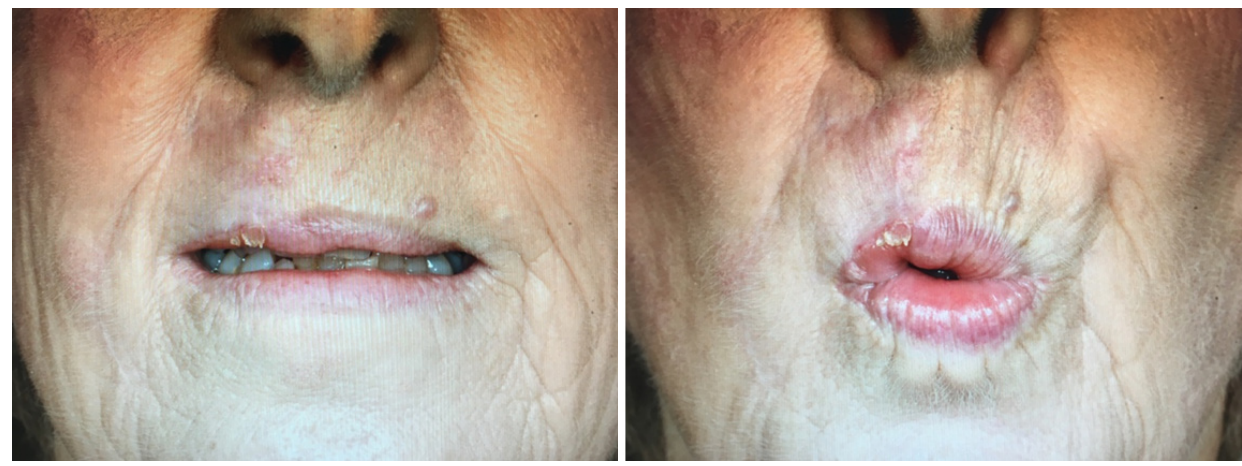

Figure 7. Images of the labio-mandibular flap at 6-months' postoperation showing excellent lip symmetry and orbicularis oris function. Note the seamless functional and aesthetic outcome

time of suture removal with no acute or sub-acute complications. Longer-term, there were no instances of microstomia while oral sphincter function was maintained in all cases. Lip symmetry was perfect in all but one case where there was slight in-curling of the lip, requiring minor secondary revision. In spite of this, all patients were satisfied with the seamless reconstruction following tumour removal. As regards completeness of oncological resection, $100 \%$ of the tumours were completely excised with follow-up at 6 months showing no signs of local recurrence.

\section{DISCUSSION}

Lip-based flap reconstructions have long been the primary like for like choice for lip defects, post-excision of skin cancers but given their associated drawbacks viz. microstomia and the need for a two-stage procedure, alternatives have been looked at. These include those based on the classical principles of local flaps e.g. the double rhomboid flaps ${ }^{[3]}$ and variants of the Webster-Barnard flap ${ }^{[4]}$. The former option is designed for commissural defects while choice of the latter would still subject the patient to difficult mouth opening.

The advent of perforator flap know-how has provided a plethora of potential perforator flaps ${ }^{[5]}$. One such example is the modiolus perforator flap described by Gunnarsson and Thomsen ${ }^{[6]}$ wherein they describe the use of a musculocutaneous perforator in a window of soft tissue bounded superiorly by the zygomaticus major, inferiorly by the risorius, medially by the oral commissure and laterally, by the facial artery. This consistent perforating vessel has been described by other studies ${ }^{[7]}$ and is the source perforator used for the perforator-plus labio-mandibular flap. However, one of the issues with the use of propeller or islanded flaps is the later onset of lymphoedema; an unaesthetic feature.

In order to overcome this, we have combined the best elements of the perforator concept and placed them within the confines of traditional aesthetic sub-unit, which in this case, refers to the labio-mandibular area. Placing the flap margins within these anatomical boundaries allows for the appearance of a scarless technique. In addition, the preservation of a cutaneous bridge preserves lymphatic channels and minimises lymphoedema. However, as the flap is rotated and advanced into the defect, the oral commissure is blunted. A "Y-V" advancement of the oral commissure is then performed and advanced into the labio-mandibular flap at its middle third. Partially dividing the flap here does not reduce its distal perfusion as the modiolar perforator is preserved just distal to the $\mathrm{Y}-\mathrm{V}$ advancement. This allows for an aesthetic reconstruction as illustrated earlier in Figure 1.

Excising these tumours with a cuff of orbicularis oris muscle allows for completeness of cancer resection while the remaining orbicularis oris muscle is sufficient to achieve an oral sphincter seal for functional purposes. This also helps maintain the dimensions of the oral aperture, negating the microstomia effect. All of these small steps contribute to an excellent functional and aesthetic outcome. 
In summary, for partial-thickness defects of upper lip and peri-commissural areas, following oncological resection, where the underlying oral mucosa is uninvolved by tumour, the perforator-plus labio-mandibular flap offers a safe and single-stage aesthetic reconstructive option with preservation of the oral sphincter and perfect lip symmetry. It must be noted that this technique is specifically suited for more superficial defects, not involving sacrifice of the lip mucosa and in particular, those in the geriatric population group.

\section{DECLARATIONS}

\section{Authors' contributions}

Manuscript writing: Kannan RY

Help in manuscript writing and editing: Nduka C

Data source and availability

Case records (Queen Victoria Hospital, East Grinstead, UK).

\section{Financial support and sponsorship}

None.

\section{Conflicts of interest}

There are no conflicts of interest.

\section{Patient consent}

Obtained.

\section{Ethics approval}

Not applicable, as this technique is based on an extension of the Labbe procedure modification (Hallam et al., 2012), developed at QVH, East Grinstead.

\section{Copyright}

(c) The Author(s) 2018.

\section{REFERENCES}

1. Kannan RY, Mathur BS. Perforator flaps of the facial artery angiosome. J Plast Reconstr Surg 2013;66:483-8.

2. Mehrotra S. Perforator-plus flaps: a new concept in traditional flap design. Plast Reconstr Surg 2007;119:590-8.

3. Andrade P, Vieira R, Brinca A, Figueirido A. Double rhomboidal flap for reconstruction of large surgical defect of the labial commissure. An Bras Dermatol 2012;87:456-8.

4. Spina P, Drummond A, Campany F, Novellino AB, Mesquita Filho J. Surgical options for the lip and perioral tumours: report of 5 cases. An Bras Dermatol 2014;89:493-6.

5. Kannan RY, Hardwicke J. Expanded version pedicled free-style perforator flaps in clinical practice: a need for a more comprehensive classification system. Eur J Plast Surg 2017; doi: 10.1007/s00238-017-1365-0.

6. Gunnarsson GL, Thomsen JB. The versatile modiolus perforator flap. Plast Reconstr Surg Glob Open 2016;4:e661.

7. Qassemyar Q, Havet E, Sinna R. Vascular basis of the facial artery perforator flap: analysis of 101 perforator territories. Plast Reconstr Surg 2012;129:421-9. 